dender Punkt der Verordnung soll die Verpflichtung zur datenschutzfreundlichen Grundeinstellung der IT sein. "Privacy by default" soll eine Verpflichtung darstellen. Die EU-Kommission verspricht sich hiervon auch wirtschaftliche Wachstumspotenziale, da dem Bürger und Verbraucher die IT-Nutzung ohne Sorgen und Bedenken vor einem Missbrauch seiner personenbezogenen Daten ermöglicht wird. Die EU-Kommission geht davon aus, dass die Bedeutung des Datenschutzes als Wettbewerbsvorteil zukünftig weiter steigen wird. Die Vorstellung der Verordnung ist für den 25. Januar 2012 geplant.

\section{BMWi: „Kompetenzzentrum Trusted Cloud“ für Cloud Computing-Forschungsprogramm}

Cloud Computing steht nicht nur bei IT-Anbietern hoch im Kurs, sondern auch auf der politischen Agenda des Bundesministeriums für Wirtschaft und Technologie (BMWi). Um deutsche Unternehmen dabei zu unterstützen, die wirtschaftlichen Potenziale von Cloud Computing zu erschließen, hat das BMWi das Technologieprogramm "Sichere Internet-Dienste - Sicheres Cloud Computing für Mittelstand und öffentlichen Sektor (Trusted Cloud)" gestartet. Begleitend zum Technologieprogramm hat das BMWi nun ein erfahrenes Expertenteam mit dem Aufbau des Kompetenzzentrums Trusted Cloud beauftragt. Unter Leitung der Management- und Technologieberatung BearingPoint (www.bearingpoint.com) werden Pierre Audoin Consultants (PAC), Public One Governance Consulting und das Hasso-Plattner-Institut das Projekt vorantreiben.

Das Ziel des BMWi Technologieprogramms ist es, die Innovations- und Marktpotenziale von Cloud Computing für mittelständische Unternehmen zu erschließen. Insgesamt 14 Forschungs- und Entwicklungsprojekte arbeiten dazu in den nächsten drei Jahren an innovativen, sicheren und rechtskonformen Cloud ComputingLösungen.

Dafür sollen Basistechnologien und anwendungsspezifische Lösungen entwickelt werden. Zugleich werden projektübergreifende Aspekte wie IT-Sicherheit, Interoperabilität, Geschäftsmodelle sowie rechtliche Fragestellungen bearbeitet.

Die Leistung des Kompetenzzentrums Trusted Cloud umfasst neben der Evaluation der Forschungsprojekte, die Begleitung des Technologietransfers, den Aufbau eines Kommunikations- und Kooperationsnetzwerks sowie die Sicherstellung der Nachhaltigkeit des Vorhabens.

Die Ergebnisse aus dem Technologieprogramm sollen dazu beitragen, wesentliche Fragen rund um den dynamischen Themenkomplex Cloud Computing zu klären, die technologische Leistungsfähigkeit von Unternehmen zu stärken und damit die Attraktivität des Wirtschaftsstandortes Deutschland zu fördern.

Für weitere Informationen: www.trusted-cloud.de

\section{Visa-Informationssystem: Bundesverwaltungsamt übernimmt Funktion der Nationalen Kopfstelle}

Am 11.10.2011 hat das europäische Visa-Informationssystem (VIS) auf Grundlage der VIS-Verordnung (Verordnung (EC) Nr. 767/2008) seinen Betrieb aufgenommen. Die Verordnung regelt Zweck, Funk- tionen, Zuständigkeiten im VIS und legt die Bedingungen und Verfahren für den Austausch von Daten über Kurzzeitvisa zwischen den Schengen-Staaten fest.

Ziel ist es, das Visumantragsverfahren zu vereinfachen und Gefahren für die Innere Sicherheit der Mitgliedstaaten zu verhindern. Das VIS soll durch einen europaweiten Datenaustausch über Visa, die für einen kurzfristigen Aufenthalt erteilt werden, dazu beitragen, Bedrohungen der öffentlichen Sicherheit vorzubeugen, Visumbetrug zu verhindern, Kontrollen an den Außengrenzen und im Hoheitsgebiet der Mitgliedsstaaten zu erleichtern sowie die Identifizierung und Rückführung illegaler Einwanderer zu ermöglichen.

Das Bundesverwaltungsamt hat in diesem Zusammenhang die zentrale Aufgabe als nationale Kopfstelle für das VIS übernommen. Es wird künftig den Betrieb sowie die laufende Wartung und Weiterentwicklung des nationalen technischen Systems wahrnehmen.

Alle zugriffsberechtigten Behörden, wie beispielsweise die Auslandsvertretungen und die Grenzbehörden, haben nun im Rahmen ihrer Befugnisse die Möglichkeit, das VIS im Laufe einer konkreten Visumvergabe zu konsultieren, z.B. wenn derselbe Antragsteller ein neues Visum beantragt.

Das VIS wird nach Abschluss der nach Weltregionen gestaffelten Inbetriebnahme alle Anträge von Schengen-Visa sowie die darauf folgenden Entscheidungen der zuständigen Visumbehörden beinhalten.

Das Verfahren wird dem Visummissbrauch vorbeugen, indem es sowohl während der Antragstellung bei den Auslandsvertretungen als auch während der Einreisekontrolle an der Grenze schnelle und sichere biometrische Verifikationen anhand von Fingerabdrücken ermöglicht.

\section{KOBIL: Apps mit Unterstützung für Hardware- Signaturen über Bluetooth Technologie}

KOBIL erweitert die mIDentity Family um fünf neue Produkte, um die neuen Bedürfnisse im Online-Banking, insbesondere im Mobile-Banking und dem Banking mit Apps auf iPhone oder Android Smartphone und Tablets, zu erfüllen.

mIDentity gilt nach wie vor als eines der sichersten und vor allem bequemsten Methoden um Online-Banking von jedem Ort der Welt an jedem PC der Welt, dank seiner „Zero Footprint Technologie", gehärtetem On-Board Browser und weltweit erstem intelligenten Security Smart Management Server (SSMS), durchzuführen. Die integrierte Update-Technologie ermöglicht dem mIDentity zu jeder Zeit auf heute noch unbekannte Gefahren zu reagieren.

Das Banking Verhalten hat sich in den letzten Jahren stark verändert. Neben dem PC basierten Internet-Banking, werden immer öfter Smartphones und Tablets, wie Apples iPad oder Android, für das Online-Banking genutzt. Dies stellt die Banken vor neue Herausforderungen in Puncto Sicherheit, zumal sich Schadsoftware auch auf diesen neuen Plattformen ausbreitet. Andere weit verbreitete Sicherheitsverfahren wie SMS-TAN können auf diesen Plattformen aufgrund der fehlenden Kanaltrennung aus Sicherheitsgründen nicht eingesetzt werden.

Deshalb erweitert KOBIL die bekannte mIDentity Linie um neue Produkte, die nun auch dem Mobile-Banking Kunden ermöglichen, Mobile-Banking nicht nur auf höchstem Sicherheitsniveau durchzuführen, sondern auch ohne Verlust von Benutzerfreundlichkeit. 